\title{
Biofeedback eletromiográfico como coadjuvante pode ajudar a manter os resultados da terapia profilática de deglutição em longo prazo na doença de Parkinson? Um estudo piloto
}

\author{
Can adjunctive electromyographic biofeedback help maintain long-
}

term prophylactic swallowing therapy results in Parkinson's disease?

A pilot study

\author{
Marcela Maria Alves da Silva Arone ${ }^{1}$ (D), Alcione Ghedini Brasolotto² (D), Gabriele Ramos de Luccas² (D), \\ Marina Gatti² (D), Claudia Tiemi Mituuti ${ }^{3}$ (D), Giédre Berretin-Felix² (1)
}

\begin{abstract}
RESUMO
Objetivo: Este estudo piloto teve como objetivo verificar a influência do uso do biofeedback EMG como método coadjuvante para auxiliar na manutenção dos resultados a longo prazo da terapia da deglutição em idosos com doença de Parkinson em uma abordagem profilática. Métodos: Os sujeitos foram avaliados quanto ao nível de ingestão oral (Functional Oral Intake Scale - FOIS), qualidade de vida (questionário SWAL-QOL) e videofluoroscopia da deglutição das consistências sólida, pudim e líquida. A gravidade da disfagia foi avaliada por meio do Dysphagia Outcome and Severity Scale (DOSS). Todos os procedimentos foram realizados antes, após três meses e após seis meses do tratamento fonoaudiológico para disfagia orofaríngea. Resultados: Três sujeitos foram tratados com terapia fonoaudiológica profilática e três com terapia convencional fonoaudiológica profilática utilizando biofeedback EMG coadjuvante em um total de 18 sessões. Seis pacientes apresentaram melhora nos níveis de ingestão oral, gravidade da disfagia e qualidade de vida após o programa de reabilitação. O nível de ingestão oral foi mantido seis meses após a terapia convencional para dois pacientes e todos os participantes tratados com biofeedback EMG. Conclusão: Ambas as modalidades de terapia profilática mostraram melhora na qualidade de vida, nível de ingestão oral e gravidade da disfagia, mas os benefícios foram mantidos ao longo do tempo apenas para os participantes do grupo experimental.
\end{abstract}

Palavras-chave: Transtornos de deglutição; Doença de Parkinson; Reabilitação; Terapia; Eletromiografia

\begin{abstract}
Purpose: This pilot study aimed to verify the influence of using EMG biofeedback as an assisting method to maintain long-term results for swallowing therapy in older adults with Parkinson's disease in a prophylactic approach. Methods: Subjects were evaluated as to the oral intake level (Functional Oral Intake Scale - FOIS), quality of life (SWAL-QOL questionnaire), and videofluoroscopy of swallowing of solid, pudding and liquid consistencies. The severity of dysphagia was assessed using the Dysphagia Outcome and Severity Scale (DOSS). All procedures were performed before, after three months, and after six months of speech-language therapy treatment for oropharyngeal dysphagia. Results: Three subjects were treated with prophylactic speech-language therapy, and three with prophylactic speechlanguage conventional therapy using adjunctive EMG biofeedback in a total of 18 sessions. Six patients experienced a reduction of the severity of the dysphagia, improved oral intake levels and quality of life after the rehabilitation program. The oral intake level was maintained six months after conventional therapy for two patients and for all participants treated with EMG biofeedback. Conclusion: Both therapy modalities with PSLT showed improved quality of life, oral intake level, and reduction of the severity of dysphagia, but the benefits were maintained over time only for participants in the experimental group.
\end{abstract}

Keywords: Deglutition Disorders; Parkinson disease; Rehabilitation; Therapy; Electromyography

\footnotetext{
Trabalho realizado na Faculdade de Odontologia de Bauru - FOB, Universidade de São Paulo - USP - Bauru (SP), Brasil.

${ }^{1}$ Speech-Language Pathology Sector, Hospital de Base de Bauru - Bauru (SP), Brasil.

${ }^{2}$ Department of Speech-Language Pathology and Audiology, Bauru School of Dentistry, Faculdade de Odontologia de Bauru - FOB, Universidade de São Paulo - USP - Bauru (SP), Brasil.

${ }^{3}$ Department of Speech-Language Pathology, Universidade Federal de Santa Catarina - UFSC - Florianópolis (SC), Brasil.

Conflito de interesses: Não.

Contribuição dos autores: MMASA: concepção e desenho do estudo, coleta, análise e interpretação dos dados, redação do artigo e aprovação final da versão a ser publicada; AGB: desenho do estudo, análise dos dados, revisão do artigo de forma intelectualmente importante e aprovação final da versão a ser publicada; GRL: redação do artigo e aprovação final da versão a ser publicada; MG: revisão do artigo de forma intelectualmente importante e aprovação final da versão a ser publicada; CTM: desenho do estudo, análise dos dados, revisão do artigo de forma intelectualmente importante e aprovação final da versão a ser publicada; GBF: concepção e desenho do estudo, análise dos dados, revisão do artigo de forma intelectualmente importante e aprovação final da versão a ser publicada. Financiamento: Coordination for the Improvement of Higher Education Personnel (CAPES).

Autor correspondente: Marina Gatti. E-mail: marina.gatti@usp.br

Recebido: Julho 08, 2021; Aceito: Agosto 17, 2021
} 


\section{INTRODUÇÃO}

A doença de Parkinson (DP) é uma patologia crônica, progressiva e degenerativa causada pela redução da produção de dopamina, sendo o envelhecimento um fator de risco ${ }^{(1)}$. A disfagia é uma das principais manifestações da DP, comumente relacionada à mastigação ineficaz ${ }^{(2)}$, função da língua prejudicada ${ }^{(3)}$, redução da elevação e excursão do complexo hiolaríngeo ${ }^{(4)}$, alteração da fase esofágica da deglutição ${ }^{(5)}$, e incoordenação entre respiração e deglutição ${ }^{(6)}$. Se não tratada, a disfagia pode comprometer o estado nutricional ${ }^{(7)}$, a qualidade de $\operatorname{vida}^{(8)} \mathrm{e}$ até levar à óbito nos casos mais graves devido ao alto risco de aspiração $0^{(9)}$.

Os sinais e sintomas da disfagia ocorrem nas fases mais avançadas da doença ${ }^{(10)}$; entretanto, uma abordagem preventiva e terapêutica pode evitar as consequências de sua evolução. Embora os estudos com abordagens profiláticas sejam escassos em doenças neurogênicas, estes têm sido frequentemente abordados nos casos de câncer de cabeça e pescoço, como forma de evitar as alterações fisiológicas da deglutição que ocorrem devido aos processos de radioterapia, com resultados favoráveis para a função de deglutição ${ }^{(11,12)}$.

No que diz respeito às estratégias de reabilitação da função da deglutição na DP, a fonoterapia tem apresentado resultados eficazes para reduzir os sinais e sintomas da disfagia, incluindo exercícios motores orais, treinamento de força muscular expiratória, técnicas posturais, tratamento vocal de Lee Silverman, compensação postural e manobras de proteção das vias aéreas ${ }^{(13)}$. Atualmente, os fonoaudiólogos estão preocupados principalmente em garantir a possibilidade de manter os resultados da terapia em longo prazo, uma vez que a DP é uma doença degenerativa e progressiva. $\mathrm{O}$ uso de terapias adjuvantes para complementar a terapia tradicional é uma possível estratégia que pode ajudar a manter os resultados, uma vez que essa abordagem visa auxiliar e otimizar o processo terapêutico.

O biofeedback eletromiográfico (EMG) é uma das estratégias terapêuticas direcionadas para a reabilitação da disfagia orofaríngea, pois melhora a força de deglutição e a coordenação muscular envolvida neste processo, associada simultaneamente à atenção das funções corticais ${ }^{(14)}$. Por ser um exame objetivo, a técnica fornece informações fisiológicas quantitativas e qualitativas, gerando imagens visuais informativas que auxiliam na compreensão do músculo treinado, facilitando o processo de reabilitação ${ }^{(15)}$.

O biofeedback EMG na disfagia neurogênica tem sido usado em casos de acidente vascular cerebral (AVC) ${ }^{(16)}$, doença de Alzheimer ${ }^{(17)}$, e esclerose lateral amiotrófica ${ }^{(18)}$, apresentando melhora da função de deglutição e redução da incidência de resultados adversos. Uma revisão sistemática recente sobre casos de DP incluiu apenas dois estudos abordando o uso de biofeedback EMG para tratar disfagia nessa população ${ }^{(19)}$. Um dos artigos mostrou melhora na biomecânica e na qualidade de vida da deglutição, indicando evidências significativas sobre a eficácia dessa abordagem para reabilitar a função alterada da deglutição em indivíduos com $\mathrm{DP}^{(20)}$. A outra se refere à tese que originou os dados deste artigo.

Uma vez que o biofeedback EMG mostrou resultados positivos para melhorar a reabilitação da disfagia orofaríngea em casos neurogênicos, os benefícios da técnica poderiam ajudar a manter os resultados pós-terapia em longo prazo para idosos com DP. Não foram encontrados na literatura estudos aplicando essa técnica em sujeitos com DP em avaliação de longo prazo.

Assim, este estudo piloto teve como objetivo verificar a influência do uso do biofeedback EMG como método auxiliar na manutenção dos resultados da terapia da deglutição em longo prazo para idosos com doença de Parkinson em uma abordagem profilática.

\section{MÉTODOS}

O Comitê de Ética em Pesquisa aprovou este estudo piloto prospectivo $\left(\mathrm{n}^{\circ} 141 / 2011\right)$, e todos os participantes assinaram o termo de consentimento livre e esclarecido. $\mathrm{O}$ estudo foi realizado com seis sujeitos do sexo masculino, com idades entre 64 e 83 anos (média 73,17 anos), diagnóstico de disfagia e DP, cujos dados individuais são apresentados na Tabela 1 .

Foram considerados os seguintes critérios de inclusão: diagnóstico de DP por laudo médico com pelo menos seis meses de antecedência e acompanhamento neurológico regular; disfagia confirmada por exame instrumental (videofluoroscopia ou nasolaringoscopia); boa saúde oral com dentes naturais ou próteses totais ou parciais com boa retenção e estabilidade; e pontuação no Mini Exame do Estado Mental ${ }^{(21)}$ compatível com o nível de escolaridade. Os critérios de exclusão foram outros distúrbios neurológicos ou câncer, ou não cumprimento do programa terapêutico proposto.

De acordo com os sinais de disfagia orofaríngea encontrados pela videofluoroscopia, os participantes foram divididos em dois grupos semelhantes quanto ao grau de disfagia (Grupo Controle $=\mathrm{GC}$; Grupo Experimental $=\mathrm{GE}$ ). Três sujeitos foram submetidos apenas à terapia fonoaudiológica profilática (TFP) (GC) e os outros três foram submetidos a TFP associada ao biofeedback EMG (GE).

A avaliação pré-terapia foi concluída entre sete e dez dias antes do processo de intervenção fonoaudiológica, e a reavaliação foi realizada entre três e seis meses após o término do

Tabela 1. Casuística segundo sexo, faixa etária, estágio da doença de Parkinson (HY), estado cognitivo (Mini Mental), gravidade da disfagia (DOSS) e nível de ingestão oral funcional (FOIS)

\begin{tabular}{|c|c|c|c|c|c|c|c|}
\hline Grupo & Paciente & Gênero & Idade (anos) & HY & $\begin{array}{l}\text { Mini Mental } \\
\text { (pontos) }\end{array}$ & DOSS & FOIS \\
\hline \multirow[t]{3}{*}{ GC } & 1 & $\mathrm{M}$ & 70 & 2 & 29 & 4 & 6 \\
\hline & 2 & $M$ & 73 & 3 & 34 & 5 & 7 \\
\hline & 3 & $M$ & 79 & 2 & 31 & 5 & 6 \\
\hline \multirow[t]{3}{*}{ GE } & 4 & M & 64 & 2,5 & 32 & 5 & 7 \\
\hline & 5 & M & 70 & 2,5 & 28 & 5 & 7 \\
\hline & 6 & $M$ & 83 & 2 & 29 & 4 & 6 \\
\hline
\end{tabular}

Legenda: $\mathbf{C G}=$ Grupo Controle; $\mathbf{E G}=$ Grupo Experimental; $\mathbf{M}$ = Masculino 
processo. Todos os procedimentos foram realizados nos mesmos períodos em todos os momentos (antes, três e seis meses após a reabilitação), respeitando o efeito de pico dos medicamentos prescritos para o tratamento da DP. Todos os procedimentos de avaliação foram realizados por uma fonoaudióloga experiente com os métodos necessários e cega para os grupos de pesquisa.

\section{Avaliações}

O estudo incluiu a aplicação de um questionário de qualidade de vida abordando os aspectos da deglutição e disfagia orofaríngea (SWAL-QOL $)^{(22)}$, considerando o escore geral. A ingestão oral também foi avaliada e os pacientes foram pontuados de acordo com os níveis da Functional Oral Intake Scale (FOIS) ${ }^{(23)}$.

A avaliação videofluoroscópica da deglutição foi realizada em vista lateral durante a mastigação e deglutição dos seguintes alimentos: (1) meia fatia de pão francês de $1 \mathrm{~cm}$ de espessura (sólido), (2) $10 \mathrm{ml}$ de pudim em colher de plástico média e (3) $10 \mathrm{ml}$ de água (líquido) oferecidos em uma seringa diretamente na boca do paciente, sendo que todas as consistências receberam contraste por meio do sulfato de bário Bariogel ${ }^{\circledR}$. O pão francês recebeu $5 \mathrm{ml}$ adicionais de contraste; o pudim foi preparado com $25 \mathrm{ml}$ de água filtrada, 2 gramas de bebida diet em pó de uva Clight ${ }^{\circledR}, 15 \mathrm{ml}$ de contraste e uma colher de medida de espessante alimentar Nestlé Resource Thicken Up Clear ${ }^{\circledR}$. O líquido foi preparado com $10 \mathrm{ml}$ de água filtrada e $10 \mathrm{ml}$ de contraste.

Após avaliação instrumental da deglutição por videofluoroscopia, os sujeitos foram classificados de acordo com o grau de disfunção da deglutição pela Dysphagia Outcome and Severity Scale (DOSS), que atribui os seguintes níveis: 7 (normal em todas as situações); 6 (deglutição funcional); 5 (disfagia leve); 4 (disfagia leve a moderada); 3 (disfagia moderada); 2 (disfagia moderadamente grave); 1 (disfagia grave) ${ }^{(24)}$. Conforme dito anteriormente, esses dados foram utilizados para definir a divisão do grupo de acordo com a gravidade da disfagia.

\section{Terapia fonoaudiológica profilática}

As sessões de TFP foram realizadas três vezes por semana durante cinco semanas. Após o término das 15 primeiras sessões, os sujeitos foram acompanhados no manejo da deglutição uma vez por semana durante três semanas, totalizando 18 sessões. Cada sessão de terapia durou 50 a 60 minutos, sendo aproximadamente 30 minutos de terapia indireta e 30 minutos de terapia direta. Todas as sessões foram conduzidas pela mesma fonoaudióloga, que não é a mesma que realizou os procedimentos de avaliação.

Todos os seis sujeitos foram submetidos a TFP para o tratamento da deglutição em caráter profilático, visto que todos os participantes apresentavam poucos sinais e sintomas de disfagia, o que corresponde aos estágios iniciais da doença. Os exercícios orofaciais, vocais e respiratórios foram aplicados de acordo com a necessidade de reabilitação, a partir do diagnóstico de disfagia orofaríngea. Cada sujeito realizou exercícios vocais selecionados após exame endoscópico nasofaríngeo da função de fala e testes terapêuticos, com resultado positivo ou neutro para qualidade vocal e conforto laringofaríngeo.
As estratégias terapêuticas utilizadas para cada participante deste estudo foram (1) exercícios isométricos de lábios, língua, bochecha e masseter, (2) exercícios isotônicos de lábios e língua, (3) / a / com adução laríngea, (4) / a / em baixa frequência e / i / em alta frequência, (5) emissão da sequência / mini / em alta frequência repetidamente; (6) / b / prolongado, (7) emissão de consoante fricativa surda/sonora, (8) exercício expiratório, (9) treinamento de deglutição de acordo com a estratégia descrita por Crary, Carnaby-Mann, Griher, Helseth ${ }^{(25)}$, e Mc Neill ${ }^{(26)}$ usando diferentes consistências alimentares, (10) manobra protetora de queixo para baixo e (11) manobra de Mendelsohn. As manobras foram solicitadas de acordo com a necessidade de cada sujeito, e sua eficácia foi demonstrada por meio de exame instrumental.

A TFP associada ao biofeedback EMG foi conduzida em três indivíduos com DP utilizando o aparelho de eletromiografia NeuroEDUCATOR ${ }^{\circledR} 4$ (quatro canais independentes com processamento em tempo real e largura de banda entre $10-1.000 \mathrm{~Hz}$ ). As estratégias de tratamento de biofeedback EMG tiveram como objetivo alcançar os ajustes neuromusculares para realizar uma deglutição funcional com registro eletromiográfico do músculo supra-hióideo. Para tanto, em cada sessão, os eletrodos foram posicionados nos músculos masseter direito e esquerdo, orbicular da boca e supra-hióideo, paralelos às fibras musculares a uma distância de $2 \mathrm{~cm}$. Os sujeitos estavam sentados, e a área de contato pele-eletrodo havia sido previamente limpa com algodão embebido em álcool ( $\left.70^{\circ} \mathrm{GL}\right)$ para remover o excesso de oleosidade e melhorar a fixação e a captação do sinal. Potenciais bioelétricos musculares foram capturados através de eletrodos de superfície usando uma fita condutora $3 \mathrm{M}$.

Durante o treinamento, foi mostrado aos sujeitos seu padrão eletromiográfico de deglutição e o padrão a ser alcançado, estabelecendo um traçado alvo para o treinamento funcional de cada paciente (Figura 1). Nesse treinamento, os pacientes realizavam as estratégias facilitadoras da deglutição, cuja eficácia foi previamente demonstrada no exame instrumental. Inicialmente, o treinamento funcional foi realizado com saliva e, conforme a função melhorava, os alimentos eram introduzidos (pudim, sólido e líquido). $\mathrm{O}$ objetivo desse treinamento foi tornar o recrutamento neuromuscular o mais semelhante possível ao padrão de traçado alvo, que envolvia um aumento da amplitude do sinal eletromiográfico para os músculos supra-hióideos durante a função por meio de maior recrutamento muscula, melhor coordenação entre os grupos musculares envolvidos na deglutição e controle simultâneo dos músculos masseter e orbicular da boca para evitar compensações. Com isso, os pacientes puderam verificar visualmente os resultados das manobras e estratégias de deglutição utilizadas para o treinamento direto, permitindo-lhes aprender um novo padrão de deglutição. O biofeedback EMG não foi utilizado durante os exercícios motores orais e vocais.

$\mathrm{O}$ treinamento da deglutição foi realizado ao término da sessão sem apoio visual direto do paciente com o equipamento e, posteriormente, os resultados foram analisados para verificar se haviam aprendido independentemente do suporte. Os sujeitos deste estudo não realizaram exercícios em casa durante toda a intervenção. 


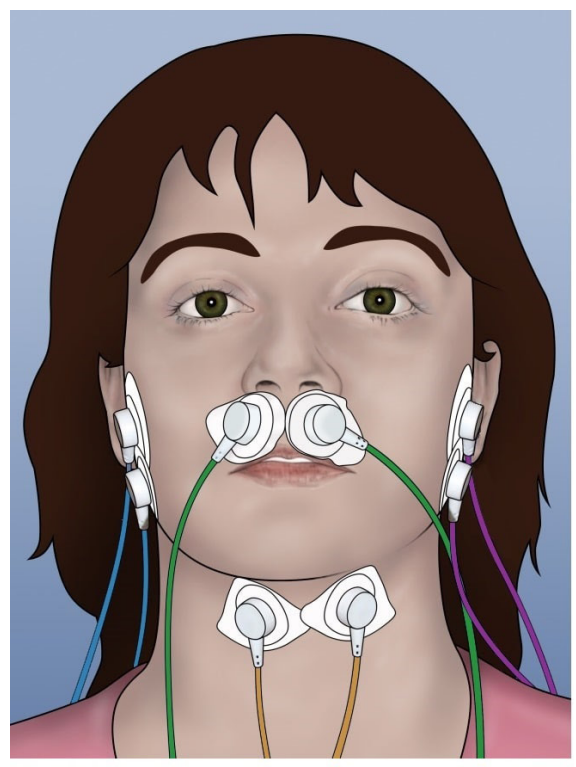

A

"uv

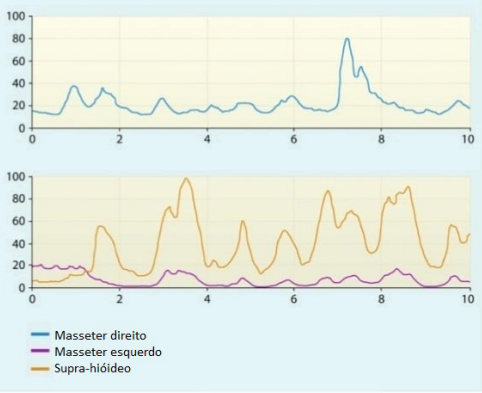

B
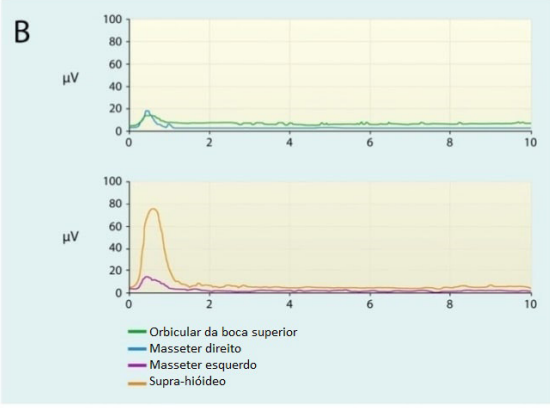

Figura 1. Posicionamento dos eletrodos e registro eletromiográfico correspondente à musculatura supra-hióidea (amarelo), músculo orbicular da boca superior (verde) e atividade masseter direita (azul) e esquerda (roxo) durante a deglutição. O gráfico A representa o padrão eletromiográfico da deglutição do paciente e o gráfico B o padrão-alvo orientado por terapia.

Tabela 2. Resultados da avaliação da qualidade de vida (SWAL-QOL), nível de ingestão oral (FOIS) e gravidade da disfagia (DOSS) dos sujeitos submetidos ao TFP e TFP associada ao biofeedback eletromiográfico

\begin{tabular}{|c|c|c|c|c|c|c|c|c|c|}
\hline \multicolumn{10}{|c|}{ TFP } \\
\hline & \multicolumn{3}{|c|}{ Caso clínico 1} & \multicolumn{3}{|c|}{ Caso clínico 2} & \multicolumn{3}{|c|}{ Caso clínico 3} \\
\hline & Pré & Pós-3 & Pós-6 & Pré & Pós-3 & Pós-6 & Pré & Pós-3 & Pós-6 \\
\hline SWAL-QOL & 60,79 & 91,48 & 79,54 & 74,43 & 84,09 & 84,09 & 72,16 & 88,07 & 84,66 \\
\hline FOIS & 6 & 7 & 7 & 7 & 7 & 7 & 6 & 7 & 6 \\
\hline Doss & 4 & 5 & 5 & 5 & 6 & 6 & 5 & 5 & 5 \\
\hline \multicolumn{10}{|c|}{ TFP associada ao biofeedback EMG } \\
\hline & \multicolumn{3}{|c|}{ Caso clínico 4} & \multicolumn{3}{|c|}{ Caso clínico 5} & \multicolumn{3}{|c|}{ Caso clínico 6} \\
\hline & Pré & Pós-3 & Pós-6 & Pré & Pós-3 & Pós-6 & Pré & Pós-3 & Pós-6 \\
\hline SWAL-QOL & 87,50 & 83,52 & 85,22 & 86,93 & 89,77 & 89,77 & 78,41 & 90,91 & 81,25 \\
\hline FOIS & 7 & 7 & 7 & 7 & 7 & 7 & 6 & 7 & 7 \\
\hline DOSS & 5 & 6 & 6 & 5 & 6 & 5 & 4 & 5 & 5 \\
\hline
\end{tabular}

Legenda: TFP = terapia fonoaudiológica profilática

\section{RESULTADOS}

TFP (CG): Os resultados mostraram melhora da qualidade de vida considerando o período antes da TFP e após três meses em todos os casos, com diminuição após seis meses nos casos 1 e 3. Houve aumento da ingestão oral após a reabilitação nos casos 1 e 3 , com os resultados sendo mantidos em todos os momentos no caso 1 e retorno ao estado anterior da intervenção no caso 3 . O caso 2 não apresentou alterações dietéticas durante o tratamento. Após a intervenção, houve melhora do grau de disfagia com resultados mantidos após três e seis meses de tratamento em dois casos (casos 1 e 2) e desempenho funcional semelhante ao observado antes do tratamento, após ambos os três meses e após seis meses no caso 3 .

TFP associada ao biofeedback EMG (EG): A análise da qualidade de vida revelou leve variação dos desfechos nos diferentes momentos estudados no caso 4 , melhora dos resultados comparando os momentos antes e três meses após a terapia, com resultados mantidos após seis meses no caso 5 e melhora considerável após três meses em relação ao período antes do tratamento no caso 6, com escores reduzidos após seis meses para este item. A ingestão oral manteve o nível máximo para os casos 4 e 5 e melhorou no caso 6, com resultados mantidos seis meses após o tratamento. Após três meses de reabilitação para os três casos, houve melhora do grau de disfagia, apresentando melhora da manutenção obtida por meio da terapia após seis meses para os casos 4 e 6 e retorno ao estado anterior à terapia após seis meses para o caso 5 .

Ambos os grupos se beneficiaram com as modalidades terapêuticas empregadas. Um participante do GC não manteve a melhora em relação ao nível de ingestão oral seis meses após o tratamento, enquanto todos os pacientes submetidos ao biofeedback EMG mantiveram os benefícios alcançados (Tabela 2).

\section{DISCUSSÃO}

Este estudo empregou estratégias comumente utilizadas para a terapia fonoaudiológica convencional da reabilitação da deglutição em casos neurogênicos. No entanto, de uma forma 
inédita, propusemos uma abordagem profilática nesta pesquisa, uma vez que todos os participantes estavam nos estágios iniciais de DP e, portanto, apresentavam sinais e sintomas precoces de disfagia. Em nossa prática clínica, os pacientes com DP frequentemente procuram o tratamento fonoaudiológico para disfagia em estágios mais avançados da doença, quando a deglutição já está gravemente prejudicada. A proposta de realização a TFP foi idealizada, pois medidas preventivas e profiláticas são importantes para evitar consequências mais graves em longo prazo nas doenças degenerativas, tendo em vista que a evolução da DP ocasiona uma piora da gravidade da disfagia $^{(10)}$. Estudos anteriores sobre câncer de cabeça e pescoço encontraram resultados positivos para melhorar ou manter aspectos como qualidade de vida e ingestão oral por meio de uma abordagem profilática antes do início do tratamento ${ }^{(11,12)}$, o que complementa os resultados desta pesquisa.

A TFP associada a estratégias convencionais aplicadas em ambos os grupos mostrou-se eficaz, pois melhorou a disfagia e o nível de ingestão oral. Isso confirma os dados de outro estudo ${ }^{(27)}$, que relatou melhora do grau de disfagia avaliada por protocolo de avaliação clínica em idosos com disfagia neurogênica tratados com exercícios miofuncionais e manobras posturais. Troche et al. ${ }^{(28)}$ encontraram melhores escores nas escalas de penetração e aspiração em indivíduos com doença de Parkinson submetidos a treinamento de força muscular expiratória. Diferentemente do nosso estudo, esses artigos realizaram avaliação pós-terapia imediatamente após a conclusão do processo terapêutico.

Além disso, em relação ao grau de disfagia, os resultados encontrados em sujeitos reabilitados por meio de terapia convencional associada ao biofeedback EMG concordam com outros achados $^{(29)}$, que estudaram o grau de disfagia em sujeitos após AVC tratados com terapia convencional associada ao biofeedback EMG e encontraram uma melhora após o tratamento.

Nenhuma investigação foi encontrada avaliando o efeito das estratégias de terapia convencional pela aplicação da Functional Oral Intake Scale (FOIS) em indivíduos com DP. Os sujeitos incluídos nesta pesquisa apresentaram resultados pré-terapia na FOIS que sugeriram uma via oral preservada. Como os pacientes já apresentavam boa ingestão oral antes das intervenções, esperavase que as estratégias mantivessem os bons resultados iniciais. Como o nível de ingestão oral melhorou ou permaneceu estável após as intervenções, entendemos que as estratégias aplicadas foram eficazes e podem ser consideradas uma forma de manter a via oral na fase inicial dos sintomas da disfagia.

Os estudos que aplicaram o biofeedback EMG em indivíduos após AVC obtiveram uma melhora significativa no nível de ingestão oral, apesar de suas amostras incluírem indivíduos com vias alternativas de alimentação ${ }^{(25,29)}$, enquanto os casos em nosso estudo estavam dentro do nível máximo definido pela escala.

A melhora na qualidade de vida após três meses de terapia convencional mostra que os benefícios relacionados à alimentação influenciaram positivamente na qualidade de vida dos participantes do estudo, semelhante aos achados de Troche et al. ${ }^{(28)}$. Este estudo mostrou uma melhora na qualidade de vida em indivíduos com DP, analisados por meio do questionário SWALQOL, mas seus resultados não foram mantidos a longo prazo. Além do caráter progressivo da doença, conforme mencionado anteriormente, devem ser consideradas as características avaliadas no questionário, que incluem habilidades de comunicação, saúde mental, funcionamento social, sono e fadiga. Embora essas habilidades sejam relacionadas à alimentação, elas podem ocorrer de forma isolada e refletir um agravamento da doença em aspectos não tratados pela terapia proposta neste estudo.

A melhora na qualidade de vida proporcionada pela fonoterapia associada ao biofeedback EMG no caso 6 está relacionada à melhora da disfagia e aumento do nível de ingestão oral, o que pode ter influenciado no alto escore da qualidade de vida alcançado após três meses de terapia. Os estudos com biofeedback EMG encontrados na literatura não investigaram a qualidade de vida, impossibilitando comparações. No entanto, deve-se considerar que a periodicidade da terapia e a necessidade de acompanhamento regular desses pacientes podem ajudar a manter os resultados em longo prazo, permitindo um maior envolvimento do paciente na intervenção, o que torna mais fácil aprender o padrão de deglutição alcançado com a terapia, bem como a automatização da função.

Em geral, os resultados alcançados pela terapia convencional foram semelhantes aos resultados obtidos pela terapia associada ao biofeedback EMG na população estudada. No entanto, devese mencionar que o uso do biofeedback EMG pode ser uma estratégia importante para manter os benefícios, uma vez que todos os participantes do GE não apresentaram piora do grau de disfagia apesar do caráter progressivo da DP. A manutenção dos resultados a longo prazo é fundamental nos casos de DP e deve ser considerada na metodologia dos programas de terapia ${ }^{(30)}$, uma vez que é uma doença progressiva e espera-se uma piora nos parâmetros da deglutição à medida que que a doença evolui ${ }^{(10)}$.

Por se tratar de um estudo de acompanhamento longitudinal, alguns fatores de interferência devem ser considerados na continuidade dos resultados, incluindo a evolução da doença, ajustes de prescrição de medicamentos e até mesmo a periodicidade da terapia. Os participantes deste estudo apresentavam casos de doença progressiva e, apesar da intervenção, a doença e os sinais e sintomas clínicos relacionados evoluíram. Dessa maneira, uma vez que os ajustes em medicação são importantes para o controle do quadro clínico, é importante considerar que essas mudanças possivelmente ocasionaram impactos no processo de reabilitação.

O estudo possui limitações relacionadas ao número reduzido de pacientes nos grupos, o que indica a necessidade de pesquisas futuras com um número maior de sujeitos. Além disso, é importante considerar, em estudos futuros, delineamentos com participantes de diferentes faixas etárias e maior gravidade das alterações na fisiologia da deglutição, o que potencializar os resultados sugeridos neste trabalho e orientar o uso de estratégias de reabilitação mais eficazes para pacientes com DP. Instrumentos de avaliação que quantificam os dados qualitativos, como tempo de trânsito oral e faríngeo ou medida da excursão do hióide, também devem ser considerados, pois permitem análises objetivas, apesar de não fornecerem informações relacionadas à deglutição de indivíduos com comprometimento leve.

\section{CONCLUSÃO}

Ambas as modalidades de terapia com a TFP resultaram em melhora da qualidade de vida, nível de ingestão oral e gravidade da disfagia, entretanto os benefícios foram mantidos ao longo do tempo apenas para os participantes do grupo experimental. 


\section{AGRADECIMENTOS}

Os autores agradecem à Coordenação de Aperfeiçoamento de Pessoal de Nível Superior (CAPES) pelo apoio financeiro e ao Grupo de Apoio ao Parkinson de Bauru pelos encaminhamentos dos pacientes. Também agradecem ao Prof. Dr. Heitor Marques Honório, pela análise estatística, e todos os pacientes que participaram deste projeto.

\section{REFERÊNCIAS}

1. Collier TJ, Kanaan NM, Kordower JH. Aging and Parkinson's disease: different sides of the same coin? Mov Disord. 2017;32(7):983-90. http://dx.doi.org/10.1002/mds.27037. PMid:28520211.

2. da Silva N, Verri E, Palinkas M, Hallak J, Regalo S, Siéssere S. Impact of Parkinson's disease on the efficiency of masticatory cycles: electromyographic analysis. Med Oral Patol Oral Cir Bucal. 2019;24(3):e314-8. http://dx.doi.org/10.4317/medoral.22841.

3. Fukuoka T, Ono T, Hori K, Wada Y, Uchiyama Y, Kasama S, et al. Tongue pressure measurement and videofluoroscopic study of swallowing in patients with Parkinson's Disease. Dysphagia. 2019;34(1):80-8. http:// dx.doi.org/10.1007/s00455-018-9916-5. PMid:29948261.

4. Kim YH, Oh BM, Jung IY, Lee JC, Lee GJ, Han TR. Spatiotemporal characteristics of swallowing in Parkinson's disease. Laryngoscope. 2015;125(2):389-95. http://dx.doi.org/10.1002/lary.24869. PMid:25093527.

5. Suttrup I, Suttrup J, Suntrup-Krueger S, Siemer ML, Bauer J, Hamacher C, et al. Esophageal dysfunction in different stages of Parkinson's disease. Neurogastroenterol Motil. 2017 Jan;29(1):e12915. http:// dx.doi.org/10.1111/nmo.12915. PMid:27477636.

6. Troche MS, Huebner I, Rosenbek JC, Okun MS, Sapienza CM. Respiratory-swallowing coordination and swallowing safety in patients with Parkinson's disease. Dysphagia. 2011;26(3):218-24. http://dx.doi. org/10.1007/s00455-010-9289-x. PMid:20623304.

7. Fávaro-Moreira NC, Krausch-Hofmann S, Matthys C, Vereecken C, Vanhauwaert E, Declercq A, et al. Risk Factors for malnutrition in older adults: a systematic review of the literature based on longitudinal data. Adv Nutr. 2016;7(3):507-22. http://dx.doi.org/10.3945/an.115.011254. PMid:27184278.

8. Chan HF, Ng ML, Kim H, Kim DY. Swallowing-related quality of life among oral-feeding Chinese patients with Parkinson's disease - a preliminary study using Chinese SWAL-QOL. Disabil Rehabil. 2020;24:1-7. http://dx.doi.org/10.1080/09638288.2020.1791979. PMid:32703037.

9. Martinez-Ramirez D, Almeida L, Giugni JC, Ahmed B, Higuchi M, Little CS, et al. Rate of aspiration pneumonia in hospitalized Parkinson's disease patients: a cross-sectional study. BMC Neurol. 2015;15(1):104. http://dx.doi.org/10.1186/s12883-015-0362-9. PMid:26141135.

10. Simons JA. Swallowing dysfunctions in Parkinson's Disease. Int Rev Neurobiol. 2017;134:1207-38. http://dx.doi.org/10.1016/ bs.irn.2017.05.026. PMid:28805570.

11. Messing BP, Ward EC, Lazarus CL, Kim M, Zhou X, Silinonte J, et al. Prophylactic swallow therapy for patients with head and neck cancer undergoing chemoradiotherapy: a randomized trial. Dysphagia. 2017;32(4):487-500. http://dx.doi.org/10.1007/s00455-017-9790-6. PMid:28444488.

12. Carmignani I, Locatello LG, Desideri I, Bonomo P, Olmetto E, Livi $\mathrm{L}$, et al. Analysis of dysphagia in advanced-stage head-and-neck cancer patients: impact on quality of life and development of a preventive swallowing treatment. Eur Arch Otorhinolaryngol. 2018;275(8):215967. http://dx.doi.org/10.1007/s00405-018-5054-9. PMid:29978259.

13. López-Liria R, Parra-Egeda J, Vega-Ramírez FA, Aguilar-Parra JM, Trigueros-Ramos R, Morales-Gázquez MJ, et al. Treatment of dysphagia in Parkinson's Disease: a systematic review. Int J Environ Res Public Health. 2020;17(11):4104. http://dx.doi.org/10.3390/ijerph17114104. PMid:32526840.

14. Humbert IA, Christopherson H, Lokhande A, German R, GonzalezFernandez M, Celnik P. Human hyolaryngeal movements show adaptive motor learning during swallowing. Dysphagia. 2013;28(2):139-45. http://dx.doi.org/10.1007/s00455-012-9422-0. PMid:22926828.

15. Crary MA, Carnaby-Mann GD, Groher ME, Helseth E. Functional benefits of dysphagia therapy using adjunctive sEMG biofeedback. Dysphagia. 2004;19(3):160-4. http://dx.doi.org/10.1007/s00455-0040003-8. PMid: 15383945.

16. Park JS, Hwang NK, Kim HH, Lee G, Jung YJ. Effect of neuromuscular electrical stimulation combined with effortful swallowing using electromyographic biofeedback on oropharyngeal swallowing function in stroke patients with dysphagia: a pilot study. Medicine (Baltimore). 2019;98(44):e17702. http://dx.doi.org/10.1097/MD.0000000000017702. PMid:31689798.

17. Tang Y, Lin X, Lin XJ, Zheng W, Zheng ZK, Lin ZM, et al. Therapeutic efficacy of neuromuscular electrical stimulation and electromyographic biofeedback on Alzheimer's disease patients with dysphagia. Medicine (Baltimore). 2017;96(36):e8008. http://dx.doi.org/10.1097/ MD.0000000000008008. PMid:28885365.

18. Tomik J, Sowula K, Ceranowicz P, Dworak M, Stolcman K. Effects of biofeedback training on esophageal peristalsis in amyotrophic lateral sclerosis patients with dysphagia. J Clin Med. 2020;9(7):2314. http:// dx.doi.org/10.3390/jcm9072314. PMid:32708232.

19. Battel I, Calvo I, Walshe M. Interventions involving biofeedback to improve swallowing in people with parkinson disease and dysphagia: a systematic review. Arch Phys Med Rehabil. 2021;102(2):314-22. http://dx.doi.org/10.1016/j.apmr.2020.06.033.

20. Athukorala RP, Jones RD, Sella O, Huckabee ML. Skill training for swallowing rehabilitation in patients with Parkinson's disease. Arch Phys Med Rehabil. 2014;95(7):1374-82. http://dx.doi.org/10.1016/j. apmr.2014.03.001. PMid:24816250.

21. Bertolucci PH, Brucki SM, Campacci SR, Juliano Y. O Mini-Exame do Estado Mental em uma população geral: impacto da escolaridade. Arq Neuropsiquiatr. 1994 Mar;52(1):1-7. http://dx.doi.org/10.1590/ S0004-282X1994000100001. PMid:8002795.

22. McHorney CA, Robbins J, Lomax K, Rosenbek JC, Chignell K, Kramer AE, et al. The SWAL-QOL and SWAL-CARE outcomes tool for oropharyngeal dysphagia in adults: III. Documentation of reliability and validity. Dysphagia. 2002;17(2):97-114. http://dx.doi. org/10.1007/s00455-001-0109-1. PMid:11956835.

23. Crary MA, Mann GD, Groher ME. Initial psychometric assessment of a funtional oral intake scale for dysphagia in stroke patients. Arch Phys Med Rehabil. 2005;86(8):1516-20. http://dx.doi.org/10.1016/j. apmr.2004.11.049. PMid:16084801.

24. O'Neil KH, Purdy M, Falk J, Gallo L. The Dysphagia Outcome and Severity Scale. Dysphagia. 1999;14(3):139-45. http://dx.doi. org/10.1007/PL00009595. PMid:10341109.

25. Crary MA, Carnaby-Mann GD, Griher ME, Helseth E. Functional benefits of dysphagia therapy using adjunctive sEMG biofeedback. Dysphagia. 2004;19(3):160-4. http://dx.doi.org/10.1007/s00455-0040003-8. PMid:15383945. 
26. Carnaby-Mann GD, Crary MA. McNeill dysphagia therapy program: a case-control study. Arch Phys Med Rehabil. 2010;91(5):743-9. http:// dx.doi.org/10.1016/j.apmr.2010.01.013. PMid:20434612.

27. Nagaya M, Kachi T, Yamada T. Effect of swallowing training on swallowing disorders in Parkinson's disease. Scand J Rehabil Med. 2000 Jun 29;32(1):115. http://dx.doi.org/10.1080/003655000750045677. PMid:10782935.

28. Troche MS, Okun MS, Rosenbek JC, Musson N, Fernandez HH, Rodriguez R, et al. Aspiration and swallowing in Parkinson disease and rehabilitation with EMST: a randomized trial. Neurology.
2010;75(21):1912-9. http://dx.doi.org/10.1212/WNL.0b013e3181 fef115. PMid:21098406.

29. Huckabee ML, Cannito MP. Outcomes of swallowing rehabilitation in chronic brainstem dysphagia: a retrospective evaluation. Dysphagia. 1999;14(2):93-109. http://dx.doi.org/10.1007/PL00009593. PMid:10028039.

30. López-Liria R, Parra-Egeda J, Vega-Ramírez FA, Aguilar-Parra JM, Trigueros-Ramos R, Morales-Gázquez MJ, et al. Treatment of dysphagia in Parkinson's Disease: a systematic review. Int J Environ Res Public Health. 2020;17(11):4104. http://dx.doi.org/10.3390/ijerph17114104. PMid:32526840. 